\title{
Atuação do enfermeiro na mudança do estilo de vida de pessoas com hipertensão: revisão narrativa da literatura
}

\author{
Nurses' performance in changing the lifestyle of people with hypertension: narrative literature \\ review
}

El desempeño de las enfermeras para cambiar el estilo de vida de las personas con hipertensión: revisión narrativa de la literatura

Recebido: 06/08/2021 | Revisado: 13/08/2021 | Aceito: 18/08/2021 | Publicado: 22/08/2021

\author{
Jailton Diniz Soares \\ ORCID: https://orcid.org/0000-0002-2592-001X \\ Universidade Regional Integrada do Alto Uruguai e das Missões-Santiago, Brasil \\ E-mail: jailton.jailton@yahoo.com.br \\ Letícia Martins Machado \\ ORCID: https://orcid.org/0000-0002-0411-1889 \\ Universidade Regional Integrada do Alto Uruguai e das Missões-Santiago, Brasil \\ E-mail: lehmachado@yahoo.com.br \\ Bárbara Belmonte Bedin \\ ORCID: https://orcid.org/0000-0003-3243-9141 \\ Universidade Regional Integrada do Alto Uruguai e das Missões-Santiago,Brasil \\ E-mail: barbarabbedin@ hotmail.com \\ Claudete Moreschi \\ ORCID: https://orcid.org/0000-0003-3328-3521 \\ Universidade Regional Integrada do Alto Uruguai e das Missões-Santiago, Brasil \\ E-mail: clau_moreschi@yahoo.com.br \\ Silvana de Oliveira Silva \\ ORCID: https://orcid.org/0000-0002-1016-0062 \\ Universidade Regional Integrada do Alto Uruguai e das Missões-Santiago, Brasil \\ E-mail: enfsilvanaoliveira18@gmail.com
}

\begin{abstract}
Resumo
O presente estudo teve como objetivo identificar as produções científicas existentes sobre a atuação dos enfermeiros na mudança do estilo de vida de pessoas com hipertensão arterial sistêmica. Trata-se de uma revisão narrativa da literatura, desenvolvida durante o mês de outubro de 2020, nas bases de dados Literatura Latino-Americana e do Caribe em Ciências da Saúde e na Base de Dados da Enfermagem, utilizando como descritores Hipertensão, Enfermagem, Estilo de vida e Educação em Saúde. Foi utilizado o operador booleano "AND”. O corpus do estudo foi constituído de 05 artigos. Após a análise qualitativa, por meio da análise temática proposta por Minayo, emergiu a seguinte categoria temática: A atuação do enfermeiro na mudança de estilo de vida de pessoas com hipertensão arterial sistêmica. Constatou-se por meio deste estudo que a responsabilidade dos enfermeiros vai além de ser o mediador entre o paciente e a hipertensão, ele é o profissional capaz de auxiliar a pessoa com hipertensão na modificação de sua vida, pois a sua proximidade e vínculo fazem com que isto seja possível. Desta forma as questões referentes a melhoria do estilo de vida, colocaram os enfermeiros como os profissionais próximo à pessoa, favorecendo sua atuação e o desenvolvimento da assistência em enfermagem.

Palavras-chave: Hipertensão; Enfermagem; Educação em saúde; Estilo de vida.
\end{abstract}

\begin{abstract}
This study aimed to identify existing scientific productions on the role of nurses in changing the lifestyle of people with systemic arterial hypertension. This is a narrative review of the literature, developed during the month of October 2020, in the Latin American and Caribbean Literature in Health Sciences and in the Nursing Database, using as descriptors Hypertension, Nursing, Style and Health Education. The Boolean operator “AND” was used. The corpus of the study consisted of 05 articles. After the qualitative analysis, through the thematic analysis proposed by Minayo, the following thematic category emerged: The role of nurses in changing the lifestyle of people with systemic arterial hypertension. It was found through this study that the responsibility of nurses goes beyond being the mediator between the patient and hypertension, he is the professional capable of helping the person with hypertension to change their life, as their proximity and bond make with make this possible. Thus, issues related to lifestyle improvement, placed nurses as professionals close to the person, favoring their performance and the development of nursing care.
\end{abstract}

Keywords: Hypertension; Nursing; Health education; Lifestyle. 


\begin{abstract}
Resumen
Este estudio tuvo como objetivo identificar las producciones científicas existentes sobre el papel de las enfermeras en el cambio de estilo de vida de las personas con hipertensión arterial sistémica. Se trata de una revisión narrativa de la literatura, desarrollada durante el mes de octubre de 2020, en la Literatura Latinoamericana y del Caribe en Ciencias de la Salud y en la Base de Datos de Enfermería, utilizando como descriptores Hipertensión, Enfermería, Estilo y Educación en Salud. El operador booleano "Y " se utilizó. El corpus del estudio estuvo conformado por 05 artículos. Tras el análisis cualitativo, a través del análisis temático propuesto por Minayo, surgió la siguiente categoría temática: El papel de las enfermeras en el cambio de estilo de vida de las personas con hipertensión arterial sistémica. A través de este estudio se encontró que la responsabilidad del enfermero va más allá de ser el mediador entre el paciente y la hipertensión, es el profesional capaz de ayudar a la persona con hipertensión a cambiar su vida, ya que su proximidad y vínculo lo hacen posible. Así, los temas relacionados con la mejora del estilo de vida, colocaron al enfermero como un profesional cercano a la persona, favoreciendo su desempeño y el desarrollo de los cuidados de enfermería.
\end{abstract}

Palabras clave: Hipertensión; Enfermería; Educación para la salud; Estilo de vida.

\title{
1. Introdução
}

As doenças crônicas não transmissíveis (DCNT) são multifatoriais, ou seja, determinadas por diversos fatores, tanto sociais ou individuais. Estas doenças são de longa duração, desenvolvidas no decorrer da vida. Responsáveis por um número alto de mortes prematuras, as DCNTs além de apresentarem elevado grau de limitação em relação às atividades laborais e de lazer, impactando financeiramente na família, pessoa e sociedade (Becker; Heidmann, 2020).

A hipertensão arterial sistêmica (HAS) é uma das DCNT mais prevalente no mundo, responsável por 13,5\% de todas as mortes, pois aumenta significativamente o risco de doenças cardiovasculares, sendo assim, é também considerada uma condição clínica multifatorial, caracterizando-se por níveis pressóricos altos do sangue (Torres et al., 2017). A pessoa com HAS, além das dificuldades que a própria doença impõe, necessitam também se adaptar-se a um novo estilo de vida, pois se trata de uma doença que requer um cuidado diário com medicação, alimentação e prática de atividade física e a partir do diagnóstico será inevitável uma busca pelo próprio bem-estar, tendo em vista diversos fatores modificáveis que farão parte de sua rotina para que obtenha assim uma qualidade de vida relacionada a sua condição de saúde (Laqui et al., 2019).

Devido aos processos de globalização e urbanização acelerados a população mundial, adotou um estilo de vida sedentário, com consumo de alimentos industrializados e de fácil preparo, mas ricos em gorduras, açúcares, sal e conservantes, diminuindo e muitas vezes substituindo as principais refeições do dia, contribuindo assim para o aparecimento das DCNT (Morais et al., 2018). A HAS destaca-se como principal causa de mortes no que se refere às doenças cardiovasculares e apresenta uma alta carga de morbimortalidade, gerando um impacto social e econômico ao interferirem na QV das pessoas, (Oliveira et al., 2019).

O conceito de QV envolve a condição humana em sua totalidade e é marcado pela subjetividade, ou seja, leva em consideração todas as dimensões que envolvem o ser humano, sejam elas psicológicas, sociais ou espirituais. Assim, dentro de um contexto comunitário ou hospitalar, possibilita uma atenção global do ser humano que está fragilizado e precisa de apoio e ajuda, com satisfação das suas necessidades diante da doença crônica (Gil; Fernandes, 2019).

O estilo de vida pode ser considerado como um fator social e ambiental da realidade em que a pessoa está inserida. Muitos destes aspectos do estilo de vida individual influenciam diretamente na rotina e na vida de cada um e são importantes, pois podem restaurar e manter a saúde (Fernandes et al., 2018).

Nesse contexto, o enfermeiro assume o papel de educador, buscando mecanismos para que a pessoa se sinta motivada em relação aos cuidados necessários para manutenção de sua saúde, sensibilizando-o sobre sua condição, além de incentivar a mudança do seu estilo de vida e hábitos saudáveis (Salles et al., 2018).

O profissional enfermeiro é um dos responsáveis por desenvolver atividades de educação em saúde, constituindo-se como uma ferramenta importante para a promoção da saúde por proporcionar conhecimento aos pacientes hipertensos, 
tornando-os ativos na transformação de vida e incentivar o autocuidado e buscara sua autonomia (Sá, et al., 2019). A educação em saúde possibilita mudanças e gera autonomia aos envolvidos, além da inclusão essencial na transformação dos fatores que impactam diretamente na vida dos pacientes, especialmente nos grupos mais vulneráveis (Geraldo et al., 2019).

O presente estudo teve como objetivo identificar as produções científicas existentes sobre a atuação do enfermeiro na mudança do estilo de vida de pessoas com hipertensão arterial sistêmica.

\section{Metodologia}

Trata-se de uma revisão narrativa da literatura. A seleção dos estudos foi norteada pela seguinte pergunta de pesquisa: O que tem sido produzido sobre a atuação do enfermeiro na melhoria do estilo de vida de pessoas com hipertensão arterial sistêmica?

A busca das produções científicas foi realizada durante o mês de outubro de 2020, nas bases de dados Literatura Latino-Americana e do Caribe em Ciências da Saúde (LILACS) e na Base de Dados da Enfermagem (BDENF), ambas acessadas a partir do formulário "Busca Avançada" do Portal Regional da Biblioteca Virtual em Saúde (BVS). Foram utilizados os descritores padronizados pelos Descritores em Ciências da Saúde: Hipertensão, Enfermagem, Estilo de Vvida e Educação em Saúde. Com o operador booleano "AND”, com a estratégia de busca "tw:((tw:(hipertensão)) AND (tw:(enfermagem)) AND (tw:(educação em saúde)) AND (tw:(estilo de vida)))".

Os critérios de inclusão foram: estudos originais e relatos de experiência realizados com adultos e/ou idosos com diagnóstico de hipertensão arterial sistêmica; publicados no idioma português, disponíveis online na íntegra. Foram excluídos as teses, dissertações e artigos nos idiomas inglês e espanhol.

Para seleção de cada artigo foi realizada a leitura do título, resumo e texto na íntegra, respectivamente. Para extração dos dados foi organizado um instrumento próprio de extração de dados que possui: código do artigo (A1, A2...), ano de publicação, periódico, país onde foi desenvolvido o estudo, objetivos, resultados e conclusões. Essa etapa foi desenvolvida por dois pesquisadores.

A busca pelas produções, representada na Figura 1, resultou na seleção de 05 artigos. Reescrever a oração anterior. Por fim, a partir do estabelecimento dos critérios de inclusão e exclusão, o corpus do estudo foi constituído de 05 artigos.

Figura 1: Fluxograma referente as buscas pelas produções.

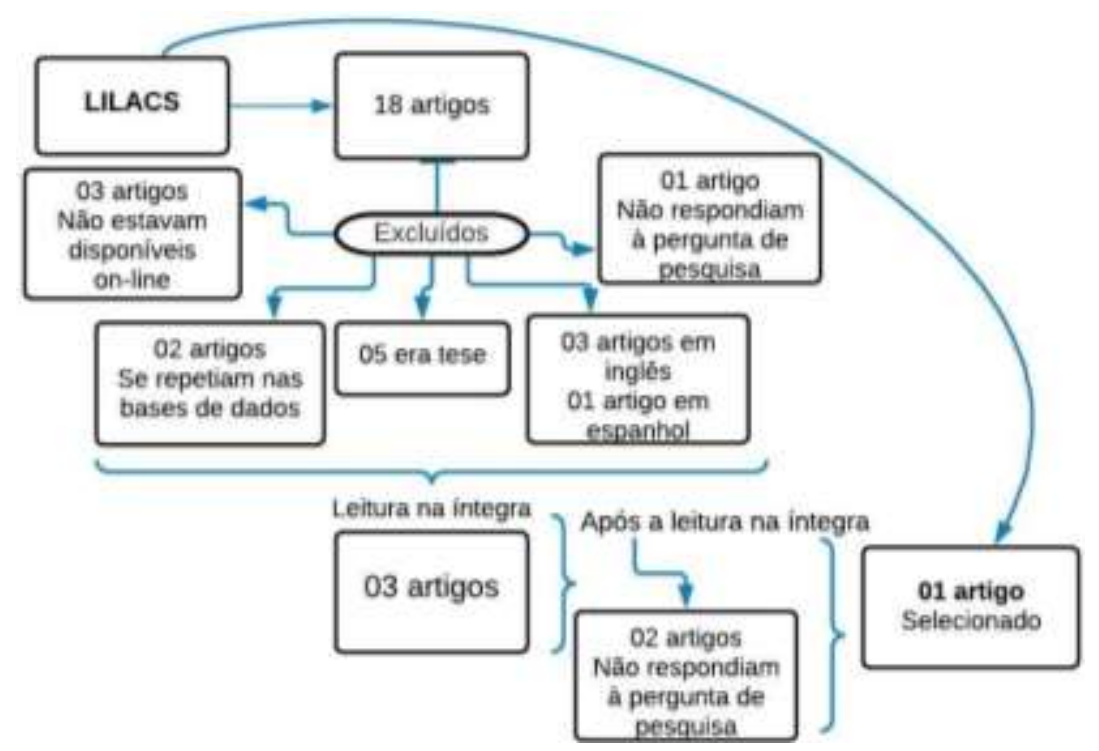




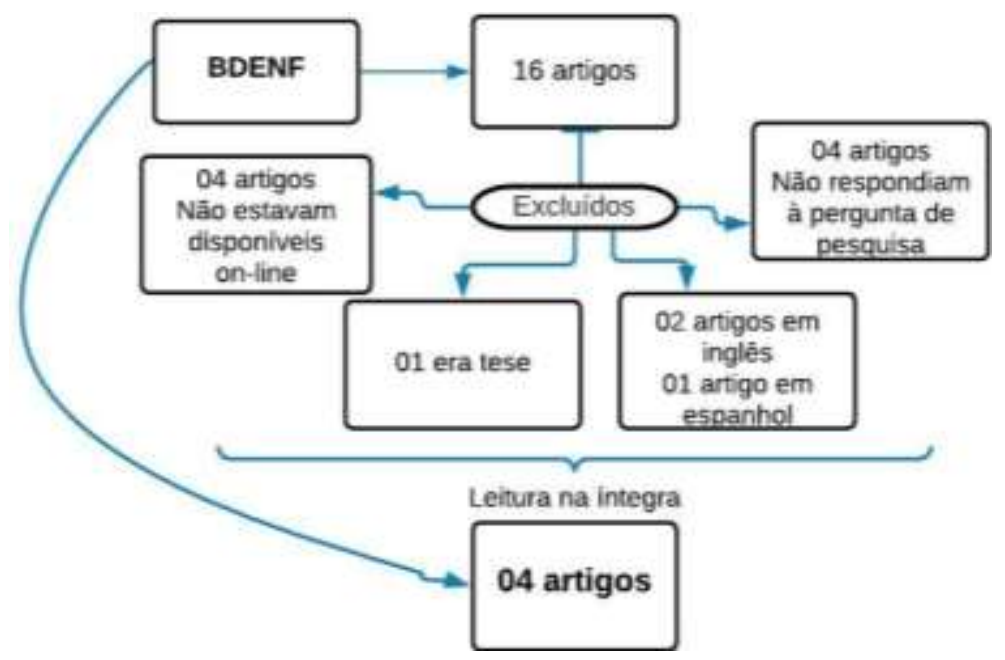

Fonte: Dados da pesquisa (2020).

A análise dos resultados seguiu a modalidade de análise temática proposta por Minayo, a qual apresenta as seguintes etapas: Pré-análise, que consiste na escolha dos artigos a serem utilizados, retomada de hipóteses e objetivos do estudo; exploração do material, que consiste na operação classificatória que visa alcançar o núcleo de compreensão do texto e tratamento dos resultados obtidos e interpretação (Minayo, 2014).

\section{Resultados}

Inicialmente foi apresentado a extração dos dados contendo as informações dos artigos selecionados, por meio do instrumento próprio de extração de dados conforme o Quadro 1. Como resultado da análise, emergiu a categoria temática: A atuação do enfermeiro na mudança de estilo de vida de pessoas com hipertensão arterial sistêmica.

Quadro 1: Instrumento para extração dos dados.

\begin{tabular}{|c|c|c|c|c|c|c|c|}
\hline $\begin{array}{l}\text { Código } \\
\text { do } \\
\text { artigo }\end{array}$ & $\begin{array}{l}\text { Ano } \\
\text { de } \\
\text { publi } \\
\text { caçã } \\
\text { o }\end{array}$ & $\begin{array}{c}\text { Periódic } \\
\text { o }\end{array}$ & $\begin{array}{c}\text { País } \\
\text { onde foi } \\
\text { desenvol } \\
\text { vido o } \\
\text { estudo }\end{array}$ & Objetivo do estudo & Resultados do estudo & Conclusões & Autor \\
\hline $\begin{array}{l}\text { A1 } \\
\text { ID: bibl } \\
\text { io- } \\
102439 \\
0\end{array}$ & 2019 & $\begin{array}{l}\text { Rev. } \\
\text { Enferm. } \\
\text { UFPE } \\
\text { On Line }\end{array}$ & Brasil & $\begin{array}{l}\text { Avaliar a qualidade de } \\
\text { vida de pessoas com hiperten } \\
\text { são arterial e relacioná- } \\
\text { la ao sexo e ao valor } \\
\text { pressórico. }\end{array}$ & $\begin{array}{l}\text { Revelou-se que a maioria } \\
\text { dos entrevistados eram } \\
\text { idosos do sexo } \\
\text { masculino, com baixa } \\
\text { escolaridade, casados e } \\
\text { pertencia à classe } \\
\text { econômica extrato da } \\
\text { Atenção Básica }\end{array}$ & $\begin{array}{l}\text { Observou-se que a } \\
\text { maioria dos } \\
\text { entrevistados apresentou } \\
\text { boa qualidade de vida. }\end{array}$ & $\begin{array}{l}\text { LAQUI, } \\
\text { Victória dos } \\
\text { Santos et al. }\end{array}$ \\
\hline $\begin{array}{c}\text { A2 } \\
\text { ID: lil- } \\
608374\end{array}$ & 2011 & $\begin{array}{l}\text { Invest. } \\
\text { Educ. } \\
\text { Enferm. }\end{array}$ & Brasil & $\begin{array}{c}\text { Descrever os estilos } \\
\text { de vida dos pacientes hiperte } \\
\text { nsos atendidos com } \\
\text { a estratégia de Saúde } \\
\text { Familiar. }\end{array}$ & $\begin{array}{l}\text { Os fatores de risco mais } \\
\text { frequentes foram } \\
\text { o sobrepeso ou obesidade } \\
\text { e a diabete mellitus A } \\
\text { qualificação do estilo de } \\
\text { vida foi } 13 \% \text { excelente, } \\
\text { muito bom } 55 \% \text {, bom } \\
27 \% \text { e regular } 4 \% \text {. }\end{array}$ & $\begin{array}{l}\text { Ainda que o estilo de } \\
\text { vida se considerou } \\
\text { satisfatório, as } \\
\text { condições e o perfil de } \\
\text { saúde indicaram a } \\
\text { persistência de fatores } \\
\text { de } \\
\text { risco de doença cardiova } \\
\text { scular. }\end{array}$ & $\begin{array}{c}\text { PAZ, } \\
\text { Elisabete } \\
\text { Pimenta } \\
\text { Araújo et al. }\end{array}$ \\
\hline
\end{tabular}




\begin{tabular}{|c|c|c|c|c|c|c|c|}
\hline $\begin{array}{c}\text { A3 } \\
\text { ID: lil- } \\
603963\end{array}$ & 2011 & $\begin{array}{l}\text { Rev. } \\
\text { Min. } \\
\text { Enferm }\end{array}$ & Brasil & $\begin{array}{l}\text { Descrever } \\
\text { a percepção dos clientes com } \\
\text { hipertensão arterial sobre } \\
\text { a consulta de enfermagem. }\end{array}$ & $\begin{array}{c}\text { Nas categorias } \\
\text { apresentadas, } \\
\text { as pessoas relataram que } \\
\text { os enfermeirosfazem o } \\
\text { seguimento } \\
\text { do tratamento } \\
\text { farmacológico dos que já } \\
\text { possuíam prescrição } \\
\text { médica prévia. }\end{array}$ & $\begin{array}{c}\text { Os } \\
\text { enfermeiros orientaram } \\
\text { sobre a importância das } \\
\text { modificações no estilo } \\
\text { de vida para } \\
\text { o controle da } \\
\text { hipertensão arterial, } \\
\text { solicitaram exames, } \\
\text { fazeram a aferição } \\
\text { da pressão arterial, } \\
\text { dentre outros aspectos. }\end{array}$ & $\begin{array}{l}\text { CARVALH } \\
\text { O, Anthonia } \\
\text { Katilianna } \\
\text { Maciel et al. }\end{array}$ \\
\hline $\begin{array}{c}\text { A4 } \\
\text { ID: lil- } \\
772193\end{array}$ & 2004 & $\begin{array}{l}\text { Rev. } \\
\text { Baiana } \\
\text { Enferm }\end{array}$ & Brasil & $\begin{array}{l}\text { Refletiu sobre a intervenção } \\
\text { da Enfermagem na prevenção } \\
\text { das doenças } \\
\text { cardiovasculares }\end{array}$ & $\begin{array}{l}\text { A inserção da prática de } \\
\text { enfermagem para a } \\
\text { prevenção das doenças } \\
\text { cardiovasculares (DCVs) } \\
\text { se faz urgente em todas } \\
\text { as estapas do } \\
\text { desenvolvimento humano }\end{array}$ & $\begin{array}{l}\text { Partido do pressuposto } \\
\text { que as DCVs tem alto } \\
\text { potencial preventivo e } \\
\text { que a enfermeira pode } \\
\text { ter uma atuação mais } \\
\text { autônoma e ampliada } \\
\text { em saúde pública }\end{array}$ & $\begin{array}{l}\text { PALMEIRA } \\
\text {, Catia } \\
\text { Suely; } \\
\text { PEREIRA, } \\
\text { Álvaro; } \\
\text { MELO, } \\
\text { Cristina. }\end{array}$ \\
\hline $\begin{array}{c}\text { A5 } \\
\text { ID: bibl } \\
\text { io- } \\
111585 \\
7\end{array}$ & 2019 & $\begin{array}{c}\text { Rev } \\
\text { Salud } \\
\text { Pública }\end{array}$ & Brasil & $\begin{array}{c}\text { Avaliou a adesão ao } \\
\text { tratamento medicamentoso e } \\
\text { não-medicamentoso de } \\
\text { usuários de um serviço } \\
\text { de atenção } \\
\text { primária diagnosticados } \\
\text { com hipertensão arterial } \\
\text { sistémica antes e após a } \\
\text { implementação da consulta } \\
\text { de enfermagem sistematizada }\end{array}$ & $\begin{array}{c}\text { Verificou-se uma } \\
\text { diferença estatisticamente } \\
\text { significativa na adesão ao } \\
\text { tratamento da hipertensão } \\
\text { arterial sistêmica após as } \\
\text { intervenções } \\
\text { de enfermagem ( } \mathrm{p}=0,102 \\
\text { ), que foram realizadas } \\
\text { individualmente e } \\
\text { coletivamente }\end{array}$ & $\begin{array}{c}\text { A assistência } \\
\text { sistematizada } \\
\text { de enfermagem pode } \\
\text { beneficiar pessoas diagn } \\
\text { osticadas } \\
\text { com hipertensão arterial } \\
\text { sistêmica na atenção } \\
\text { primária em saúde. Foi } \\
\text { identificado a } \\
\text { necessidade do } \\
\text { fortalecimento da } \\
\text { utilização do processo } \\
\text { de enfermagem e da } \\
\text { identidade do } \\
\text { enfermeiro } \\
\text { no cuidado das } \\
\text { condições crônicas }\end{array}$ & $\begin{array}{l}\text { AMARAL, } \\
\text { Beatriz et al. }\end{array}$ \\
\hline
\end{tabular}

Fonte: Dados da pesquisa (2020).

\section{Discussão}

Após a análise qualitativa dos estudos emergiu a seguinte categoria temática: A atuação do enfermeiro na mudança de estilo de vida de pessoas com hipertensão arterial sistêmica.

\section{A atuação do Enfermeiro junto a pessoas com Hipertensão Arterial Sistêmica}

Os estudos analisados identificaram que o profissional enfermeiro é visto com potencial para atuar na prevenção de doenças cardiovasculares na atenção primária, pois podem trabalhar juntamente com a comunidade e com as pessoas com HAS, levando em conta as mudanças de comportamento, hábitos e estilo de vida próprio de cada usuário (A4).

Conforme a teoria de Dorothea Orem, todos os seres humanos têm potencial para desenvolver habilidades intelectuais e práticas para o autocuidado, que, quando desenvolvidas corretamente, ajudam a manter a integridade estrutural e funcional do corpo. Assim as ações voltadas para as pessoas com HAS, devem estar pautadas no cuidado continuo, onde o indivíduo faça parte do seu cuidado, o autocuidado reflete sobre o modo de agir e ajuda na compreensão dos fatores relacionados ao paciente hipertenso e o faz ver a realidade que o rodeia e a necessidade de construir um plano de autocuidado (Teston et al., 2017).

A revisão narrativa de literatura também apontou sobre a importância do vínculo entre os enfermeiros da Estratégia 
Saúde da Família (ESF) e as pessoas com HAS. Por meio do vínculo com o usuário é possível construir projetos intervencionistas adequados às necessidades de cada usuário, favorecendo a adesão terapêutica frente ao tratamento da HAS, obtendo resultados mais significativos. Diante deste cenário faz-se necessário também as ações em saúde visando a prevenção e promoção da saúde além de mudanças nos hábitos de risco (A1). Citar conforme APA

O vínculo do usuário com o enfermeiro da ESF, inicia-se no acolhimento e em todo o processo de cuidar, iniciando na entrada do usuário ao serviço e acompanha-lo durante todo o processo do cuidado. O vínculo é capaz de ampliar a eficácia das ações de saúde, favorecer a participação e envolvimento na prestação de cuidado, no qual, o vínculo se consolida mais facilmente, identificando as necessidades dos sujeitos (Queiroz et al., 2018).

Neste contexto, o vínculo entre o enfermeiro da ESF e o usuário com HAS, possibilita a aproximação entre ambos, sendo uma ferramenta que poderá estimular as práticas de autocuidado, representando uma relação respeitosa, promovendo laços de confiança, onde o usuário poderá sanar dúvidas como por exemplo sobre os medicamentos em uso para o controle e tratamento da doença e outros assuntos relacionados à saúde, favorecendo a continuidade da assistência prestada com a participação ativa do usuário neste processo de cuidado, no qual, o mesmo tem o direito de ouvir, questionar e opinar (Rêgo; Radovanovic, 2018).

Também identificou-se no presente estudo, que o tratamento da HAS está associado às mudanças de hábitos de vida como o tabagismo, o sedentarismo, a obesidade e uma alimentação saudável e para que aconteçam essas mudanças é necessário o envolvimento dos profissionais da saúde. Nesse sentido, cabe aos enfermeiros abordarem a prevenção de doenças e promoção da saúde, prestando informações ao público alvo, pois na prática assistencial os profissionais que atuam na atenção básica exercem uma função especial no controle e tratamento da hipertensão (A2).

$\mathrm{Na}$ assistência primária ao usuário com HAS, o enfermeiro tem papel relevante no rastreamento de novos casos, prevenção e promoção da saúde através da consulta de enfermagem individual ou coletiva. Porém, se faz necessário o fortalecimento do processo de enfermagem e do autocuidado do usuário diante das condições crônicas, possuindo uma visão ampliada de promoção, prevenção e reabilitação em saúde (Amaral et al., 2019).

No contexto da atuação do profissional enfermeiro junto a pessoas com HAS, a consulta de enfermagem ainda se encontra fragilizada e fragmentada quando relacionadas aos fatores culturais da população para a mudança no estilo de vida. Os enfermeiros centram-se no controle da hipertensão arterial, a partir da aferição da pressão arterial, solicitam exames e fazem o seguimento do tratamento farmacológico daqueles que já possuíam prescrição médica prévia (A5; A3).

Diante disso, faz-se necessário a definição de padrões tornando o enfermeiro imprescindível em implementar estratégias para que sua equipe assegure aos pacientes uma assistência desejada e efetiva A padronização dos procedimentos de enfermagem é um instrumento gerencial atual e tem sido amplamente estudada pela classe e permite que todos os trabalhadores da área prestem um cuidado padronizado para o paciente de acordo com os princípios técnico-científicos, tendo também uma finalidade educativa (Salles et al., 2017).

Há centralidade na atuação médica, sem falar na gestão insuficiente da atenção primária e ausência de protocolos e capacitação da enfermagem, sendo necessário um fortalecimento e uma maior efetividade das ações de enfermagem. Apontouse que se faz necessário o fortalecimento do processo de enfermagem e do papel do enfermeiro, pois se constatou uma significativa adesão ao tratamento farmacológico e não farmacológico após as intervenções de enfermagem através da assistência sistematizada, gerando benefícios as pessoas com HAS (A5; A3).

O enfermeiro necessita atuar buscando sistematizar suas ações de intervenção salientando as mudanças de hábitos e estilo de vida, os cuidados específicos do enfermeiro são realizados durante a consulta de enfermagem, onde o foco é principalmente nas orientações das ações para reduzir a pressão, como, por exemplo, alimentação correta, reduzir o consumo de sódio e álcool, diminuir o estresse, abandonar o tabagismo e praticar atividades físicas. Essas orientações são importantes, 
pois ajuda no controle dos fatores de riscos e na realização do tratamento medicamentos (Rebelo et al., 2020).

A educação em saúde é um processo em que todos saem ganhando, o enfermeiro como educador das pessoas e como incentivador de sua equipe é o responsável em fazer com que desperte em todos a vontade de buscar novas maneiras para o enfrentamento do processo de saúde-doença e o que pode ser feito para colocá-los em prática, diante de um cenário onde todos estão envolvidos, seja o próprio enfermeiro, equipe ou pacientes e familiares (Ribeiro, 2017).

A revisão narrativa também trouxe que a ausência de protocolos e capacitações interfere na atuação dos enfermeiros junto às pessoas hipertensas. Os fatores culturais da população centralizam no médico a forma de como deve ser o acompanhamento e o tratamento das doenças, com a falta de capacitação, de protocolos que embasem e fortaleçam o atendimento e ações do enfermeiro (A5).

Além disso, a ausência de protocolos, a falta de capacitação dos profissionais enfermeiros, são fatores que influenciam na atuação dos enfermeiros junto com os usuários hipertensos. Dessa forma, acredita-se que seja necessário a implementação de protocolos, trabalhar a capacitação dos enfermeiros, baseada em uma metodologia científica que identifica os principais problemas por meio da escuta sensível, o que poderá contribuir para o enfermeiro, juntamente com a equipe, planejar intervenções que respeitam a individualidade e a sua inserção familiar, bem como é um começo para a construção da autonomia do usuário (Engela et al., 2018).

Dessa maneira, a consulta de enfermagem está ligada ao processo educativo em relação aos cuidados necessários para a manutenção da saúde do usuário. Portanto, a consulta de enfermagem torna-se um instrumento crucial de incentivo à adesão às ações na atenção básica e no acompanhamento de pacientes hipertensos, sensibilizando-os sobre suas condições de saúde e pactuando efetivamente as metas e planos de cuidado (Silva et al., 2019).

\section{Considerações Finais}

Percebeu-se por meio deste estudo que a responsabilidade do enfermeiro vai além de mediador entre o paciente e a HAS, ele é o profissional capaz de auxiliar a pessoa com HAS na modificação de sua vida, pois a sua proximidade e vínculo fazem com que isto seja possível. O enfermeiro é o profissional da saúde que tem o potencial de trazer a pessoa hipertensa para o reconhecimento e aceitação do seu momento de saúde e apresentar a ele as estratégias para uma possível melhora. Além de educador em saúde é o profissional que atenderá a pessoa hipertensa de uma maneira ampla, com uma visão alinhada a tudo o que traz consigo, este profissional analisa e avalia cada situação, com um olhar ampliado desde o início do diagnóstico de HAS.

Desta maneira, o enfermeiro acompanha o paciente desde os hábitos diários mais simples, como o preparo de refeições com pouca adição de sal, práticas de atividade física, cuidados com o peso entre outros fatores que se modificados farão com que o paciente tenha um estilo de vida mais saudável e uma melhora significativa em seu quadro clínico. Portanto, neste estudo, as questões referentes a melhoria do estilo de vida, colocam o enfermeiro como o profissional próximo à pessoa, favorecendo sua atuação e o desenvolvimento da assistência em enfermagem.

\section{Referências}

Amaral-Moreira Mota, B., Moura-Lanza, F., \& Nogueira-Cortez, D. (2019). Efetividade da consulta de enfermagem na adesão ao tratamento da hipertensão arterial sistèmica. Revista de Salud Pública, 21(3) https://doi.org/10.15446/rsap.v21n3.70291

Becker, R. M., \& Heidemann, I. T. S. B. (2020). Promoção da saúde no cuidado às pessoas com doença crônica não transmissível: revisão integrativa. Texto \& Contexto-Enfermagem, 29 DOI: https://dx.doi.org/10.1590/1980-265X-TCE-2018-0250

Engela, L. M. H. T., Rodarte, A. C., Rotondaro Júnior, A., Seixas, C. T., Viegas, S. M. D. F., \& Lanza, F. M. (2018). Uso das tecnologias em saúde na atenção básica às pessoas em condições de hipertensão arterial sistêmica. Rev Fund Care Online, 10(1), 75-84. https://doi.org/10.9789/2175-5361.2018.v10i1.75-84 
Fernandes, I. N. M., de Almeida, K. R., \& Rocha, F. C. (2018). Análise do estilo de vida dos profissionais de saúde da oncologia. Rev. enferm. UFPE on line, 2583-2589. https://doi.org/10.5205/1981-8963-v12i10a237209p2583-2589-2018

Geraldo, M.C.H.M., Corrêa, V.D.A.F., Freire, M.A.M., Dias, J.R., de Mello, A.S., \& Acioli, S. (2019). Política de educação popular: práticas na estratégia saúde da família. J Nurs UFPE on line, 13, e243335 https://doi.org/10.5205/1981-8963.2019.243335

Gil, L.M.T.S., \& Fernandes, I. M. R. (2019). Qualidade de vida da pessoa com doença inflamatória intestinal. Revista de Enfermagem Referência, 4(23), 8998. https://doi.org/10.12707/RIV19048

Girão, A. L. A., \& Freitas, C. H. A. D. (2016). Usuários hipertensos na atenção primária à saúde: acesso, vínculo e acolhimento à demanda espontânea. Revista Gaúcha de Enfermagem, 37. https://doi.org/10.1590/1983-1447.2016.02.60015

Laqui, V. D. S., Trevisan, F. G., Sanches, R. D. C. N., Rêgo, A. D. S., Paiano, M., \& Radovanovic, C. A. T. (2019). Qualidade de vida de pessoas com hipertensão arterial. Rev. enferm. UFPE on line, 1327-1337 https://doi.org/10.5205/1981-8963-v13i05a238242p1327-1326-2019

Mendes, A. J. B., de Azevedo Ponte, K. M., \& Farias, M. S. (2015). Cuidados de enfermagem para adesão, por idosos, de hábitos saudáveis de vida, com base na Teoria da Promoção da Saúde. Revista Kairós: Gerontologia, 18(4), 269-287 https://doi.org/10.23925/2176-901X.2015v18i4p269-287

MINAYO, M. C. S. O Desafio do Conhecimento: pesquisa qualitativa em saúde. 14. ed. São Paulo: Hucitec, Abrasco, 2014.

Morais, H.C.C., do Nascimento Cavalcante, S., Nascimento, L.B., Mendes, I.C., do Nascimento, K.P., \& Fonseca, R. (2018). Fatores de risco modificáveis para doenças crônicas não transmissíveis entre estudantes universitários. Rev Rene, 19, 1-8. https://doi:10.15253/2175-6783.2018193487

Nascimento, M. E.; Barbosa, E. S. (2014) Intervenções educativas voltadas para o cuidado do paciente com hipertensão arterial: Uma revisão de literatura. Revista Interfaces em Saúde. 1 (2), 9-20. https://www.fvj.br/revista/wp-content/uploads/2015/10/11_Interfaces_2014_2.pdf

Oliveira, C. D. A., Morais, H. C. C., Mendes, I. C., Silva, A. K. D., Maniva, S. J. C. D. F., \& Ferreira, P. J. D. O. (2019). Fatores de risco para doenças crônicas não transmissíveis em sacerdotes. Rev. enferm. UFPE on line, 640-646. https://doi.org/10.5205/1981-8963-v13i03a236336p640-646-2019

Palmeira, C. S., Pereira, Á., \& Melo, C. (2004). Prática de enfermagem na prevenção das doenças cardiovasculares. Revista Baiana de Enfermagem (1) 9. http://dx.doi.org/10.18471/rbe.v19i1.3893

Paz, E. P. A., do Nascimento Souza, M. H., Guimarães, R. M., Pavani, G. F., dos Santos Correa, H. F., de Carvalho, P. M., \& Rodrigues, R. M. (2011). Estilos de vida de pacientes hipertensos atendidos com a Estratégia de Saúde Familiar. Investigación y Educación en Enfermería, 29(3), 467-476. https://www.redalyc.org/articulo.oa?id=105222406016

Queiroz, R.F.D., Alvarez, A. M., Morais, L. J., \& Silva, R.A. R.D. (2019). Percepção de trabalhadores de enfermagem sobre o cuidado ao idoso portador de hipertensão arterial sistêmica. Revista Brasileira de Enfermagem, 72, 3-13. http://dx.doi.org/10.1590/0034-7167-2016-0681

Rabelo, L. M., Alexandre, K. V., Celestino, M. S., Cangirana, J. F., Albuquerque, L. K. A., Soares, S. M. L. P., \& Costa, J. S. (2020). Papel do enfermeiro na prevenção da hipertensão arterial sistêmica em idosos. Revista Brasileira de Pesquisa em Ciências da Saúde, 6(12), 22-28 10.6084/m9.figshare.11874123

Rêgo, A.D.S., \& Radovanovic, C.A.T. (2018). Adesão/vínculo de pessoas com hipertensão arterial na Estratégia Saúde da Família. Revista Brasileira de Enfermagem, 71, 1030-103. https://doi.org/10.1590/0034-7167-2017-0297

Ribeiro, M. B. (2017). A educação permanente no treinamento do enfermeiro de centro cirúrgico: revisão integrativa. Rev. SOBECC, 98-105 https://revista.sobecc.org.br/sobecc/article/view/173

Sá, G. G. D. M., Silva, F. L., Santos, A. M. R. D., Nolêto, J. D. S., Gouveia, M. T. D. O., \& Nogueira, L. T. (2019). Tecnologias desenvolvidas para a educação em saúde de idosos na comunidade: revisão integrativa da literatura. Revista latino-americana de enfermagem, 27 https://doi.org/10.1590/15188345.3171 .3186

Salles, A. L. D. O., Sampaio, C. E. P., Pereira, L. D. S., Malheiros, N. S., \& Gonçalves, R. A. (2019). O enfermeiro e a questão da adesão do paciente ao tratamento da hipertensão arterial sistêmica. Rev. enferm. UERJ, e37193-e37193 http://dx.doi.org/10.12957/reuerj.2019.37193

Silva, A., dos Santos, E. A., Feitoza, J. E. A., de Souza Melo, M. L., de Oliveira, R. D. S. A., Caldas, S. S., ... \& Medeiros, K. M. F. (2019). estratégia de educação em saúde para a adesão de hipertensos à consulta de enfermagem na atenção básica. Revista Interfaces: Saúde, Humanas e Tecnologia, 7(1), 203209. http://interfaces.leaosampaio.edu.br/index.php/revista-interfaces/article/view/659/pdf

Silva, F. V. F. D., Silva, L. D. F. D., Guedes, M. V. C., Moreira, T. M. M., Rabelo, A. C. S., \& Ponte, K. M. D. A. (2013). Cuidado de enfermagem a pessoas com hipertensão fundamentado na teoria de Parse. Escola Anna Nery, 17, 111-119 https://doi.org/10.1590/S1414-81452013000100016

Teston, E. F., Sales, C. A., \& Marcon, S. S. (2017). Perspectivas de indivíduos com diabetes sobre autocuidado: contribuições para assistência. Escola Anna Nery, 21 https://doi.org/10.5935/1414-8145.20170043

Torres, G. M. C., Figueiredo, I. D. T., Cândido, J. A. B., Pinto, A. G. A., Morais, A. P. P., Araújo, M. F. M., \& Almeida, M. I. D. (2018). Comunicação terapêutica na interação profissional de saúde e hipertenso na estratégia saúde da família. Revista Gaúcha de Enfermagem, 38. https://doi.org/10.1590/19831447.2017.04.2016-0066 